\title{
Factores de Predicción de Recidivas en Niños con Crisis Epilépticas tratadas por cinco años
}

\author{
Dr. Marcelo Devilat B.; Dra. Laura Germaín P.; Dr. Bolívar Valenzuela M. \\ Prediction of Relapses After Five Years of \\ Treatment in Children with Epileptic Seizures
}

This study examines some factors potentially predictive of relapses in a group of children with epileptic seizures. The most significant findings corcemed children who had experienced 2 or more seizuses during treatment, and those with cognitive disorders or behavioral problems. These two groups had significantly greater frecuency of relapses in comparison to children without that problems. Other factors studied apparently do not affect the incidence of relapse. Among these variables are age, sex and family history, a finding consistent with those of other authors. A third group of factors (including mixed seixures and epilepsy) was associated with a trend toward greater frecuency of relapses, howerer this trend did not reach statisfical signifieance in contrast to other findings reported in the literature.

La duración del tratamiento anticonvulsivo varía entre 1 y 5 años, a pesar de lo cual, aproximadamente el $30 \%$ de los niños vuelve a tener crisis

Servicio de Neurología y Psiquiatría. Hospital Luis Calvo Mackenna Sede Oriente. Universidad de Chile.

NOTA: Aparte de los autores, el cuidado de los niños de esta investigación estuvo a cargo de los doctores Javier Cox, Francisco Mena, Alicia Valdivieso y Rodrigo Chamorro. Los autores agradecen al Dr. Ricardo Erazo su valiosa cooperación en una parte de este trabajo. después que la medicación ha sido retirada ${ }^{1}$.

Ya que no todos los enfermos tienen las mismas caracteristicas y que no es indjferente tratar a un nif̂́o por un año o por cinco ${ }^{1}$, el análisis de las variables que pueden predecir las recaidas reviste especial importancia. Por otra parte, si dichas caracteristicas predictivas pueden ser aisladas, la longitud del tratamiento debiera ser de acuerdo al riesgo de recaer que tengan los pacientes.

El objetivo de esta investigación es estudiar los 
factores que predicen las recaídas en los niños con crisis convulsivas, después de la suspensión de los medicamentos, así como identificar las características de los pacientes que no están en relación con dichas recaídas. A la luz de los resultados obtenidos, se hará una revisión de la literatura disponible.

\section{MATERIAL Y METODOS}

En una publicación anterior ${ }^{1}$ fue descrito en detalle el material y los métodos utilizados en el análisis de 91 niños con crisis convulsivas tratados por 5 años y observados por 3 a partir del inicio de la suspensión del medicamento. Algunas características de esa investigación 1 que no fueron mencionadas previamente, se comunican aqui.

Por no disponer de facilidades, en los años en que los pacientes fueron tratados, para medir concentraciones en el plasma de anticonvulsivantes, sólo en 5 niños se realizó dicho examen.

De 20 enfermos que volvieron a tener crisis durante el tratamiento, 17 recibieron 2 o 3 fármacos. El resto sólo requirió ajuste de la dosis.

Se entenderá aqui por enfermos orgánicos a aquellos que presentaron una o más de las sigujentes caracteristicas: retardo mental (CI menor de 70), trastorno específico del aprendizaje y antecedentes de daño pre, peri o postnatal. 22 enfermos fueron orgánicos, 64 no lo eran y en 5 dicha información fue dudosa.

Se entenderá como crisis única a una descarga de origen neuronal, paroxística y sincrónica que clínicamente se expresa como una alteración de la conciencia, de la experiencia o ambas, con sintomas motores o sensoriales y que ocurre una sola vez en un paciente. Si dichos episodios ocurren más de una vez se hablará de epilepsia. Si las crisis se asocian a una enfermedad aguda, -por ejemplo, crisis febriles- se les llamará crisis ocasionales.

Se definió como trastorno cognitivo y de la conducta a las siguientes alteraciones: trastorno específico del aprendizaje o Sindrome Hiperquinético o trastorno conductal franco (cleptomania, fuga del hogar, etc.). 29 niños tuvieron 10 más de las características señaladas y 62 ninguna.

70 enfermos tenían un electroencefalograma tomado antes de la suspensión del medicamento, el que se realizó en el Instituto de Neurocirugia de Santiago (Dres. N. Chiofalo y A. Fuentes). El examen fue realizado en vigilia en todos los enfermos menos en uno. Como medio de activación se usó hiperventilación en 66 y no se efectuó en 4.

30 pacientes tuvieron el EEG anormal al momento de iniciar la suspensión de los medicamen- tos, el que fue definido como aquel que tenía actividad focal lenta (theta o delta) o irritativa o mixta (lenta e irritativa). Como anormal se catalogaron también aquellos EEG que mostraron actividad paroxística generalizada (lenta o irritativa o mixta. La ocurrencia de dichas anormalidades sucedieron en reposo o en hiperventilación.

Por último, ninguno de los enfermos que recayeron ingerian fármacos potencialmente convulsivantes al momento de recaer.

\section{RESULTADOS}

Sexo y antecedente de epilepsia en la Familia: No se observó diferencia importante entre hombres y mujeres con respecto a las recaídas, puesto que 14 de 50 niños ( $28 \%$ ) recayeron y 10 de 41 niñas $(24,4 \%)$ tuvieron esa misma evolución. A su vez de 39 enfermos que tenian entre sus familiares de primer grado, alguno con epilepsia, volvieron a tener crisis $11(28,2 \%)$, en tanto que aconteció eso en $12(25,5 \%)$ de 47 pacientes que no tenían epilépticos en sus familias. En 5 niños no se pudo obtener la información de epilepsia familiar. Ninguna diferencia se observa entonces entre ambos grupos de niños.

Edad de comienzo de las convulsiones: Los pacientes fueron divididos en dos grupos de acuerdo al inicio de sus crisis; desde después del período de recién nacido hasta los dos años de vida (50 niños) y desde los tres años a los once (41 pacientes). Los respectivos porcentajes de recaídas fueron $22 \%$ (11 enfermos) y $31,7 \%$ ( 13 nixos). Aunque se aprecia una mayor tendencia a recaer en los niños mayores, la diferencia no result 6 significativa $(x 2=2.42 \mathrm{p}>0.05)$. Por otta parte, de los 10 niños menores de un año que conformaban el grupo, recayeron 2 y 8 no lo hicieron. Se observa asi que los lactantes menores no recaen más frecuentemente que en el grupo total.

Forna de la Crisis: Ninguno de 4 enfermos con crisis ocasionales recayó. A su vez el $29,5 \% 18$ de 61 niños portadores de epilepsia volvió a presentar crisis, en tanto que el $23 \%$ de 26 pacientes que tuvieron una crisis previa al tratamiento lo hizo. La diferencia entre los dos últimos grupos no es significativa $(\times 2=0,37 p>0,05)$.

Tipo de Crisis: De 75 niños con crisis generalizadas recayeron $19(25,3 \%)$. A pesar que ningún enfermo con crisis focales volvió a presentar episodios, de 13 pacientes que tenian crisis generalizadas y focales recayó un $38,5 \%$, es decir 5 niños. Si bien es cierto que existe una mayor tendencia a las recaídas de este último grupo, la diferencia no es significativa $\left(\mathrm{x}_{2}=2.06 \mathrm{p}>0.05\right)$.

Organicidad: $E 127,3 \%{ }^{6}$ de 22 enfermos orgá. nicos recayó en tanto que el $26,6 \%{ }^{17}$ de 64 pa- 
cientes no orgánicos volvió a tener crisis. Aparece claro, que no hay relación entre organicidad y recajidas. En 5, el factor orgánico no pudo ser aclarado.

Severidad de las Crisis: Antes de iniciar el tratamiento, 54 nitos presentaron de 1 a 2 crisis. De este grupo, recayeron $13(24,1 \%)$. A su vez 26 enfermos tuvicron 3 o más episodios. De ellos, 7 $(26,9 \%)$ volvieron a tener crisis. No se pudo obtener información en 10 niños y de este análisis fue excluido un enfermo portador de ausencias sim. ples. No se observó diferencia significativa $\left(x_{2}\right)=$ $2.20 \mathrm{p}>0.05$ ) entre los dos grupos estudiados Por otra parte, durante el tratamiento (Tabla 1). 69 pacientes no tuvieron nuevas crisis, a pesar que $16(23,2 \%)$ recayeron al final de él. 15 niños presentaron 2 o más episodios en tratamiento, de estos, $7(46,7 \%)$ recayeron. En dos enfermos, la información correspondiente no fue posible de obtener. $Y$ de 5 pacientes que presentaron una crisis durante el tratamiento, ninguno recayó. Se observa que cl hecho de tener 2 o más episodios durante el tratamiento es un factor predictivo do recaidas $\left(x_{2}=4.20 p<0.05\right)$.

Tabla I.

91 Niños con Crisis Epilépticas

Reeuidas y frecuencia de crisis durante el trar.umicnto

\begin{tabular}{|c|c|c|}
\hline & Recaitas \% & No recaídas \% \\
\hline OCrìsis & $1623.2+$ & 76.8 \\
\hline 1 Crisis & - & 5 \\
\hline $2 \dot{0}+$ Cris. & $746.7+$ & 53.3 \\
\hline No datos & 1 & I \\
\hline Total & $\begin{array}{l}24 \\
(+1) x=4.20\end{array}$ & $\begin{array}{l}67 \\
p<0.025\end{array}$ \\
\hline
\end{tabular}

Trastornos Cognitivos y de la Conducta: el $37,9 \% 11$ de 29 niños que presentaron una alteración (Tabla 2) volvieran a tener crisis, en tanto que el $21 \% 1^{3}$ de 62 pacientes que no tuvieron esas alteraciones, recayó. La diferencia entre annbos grupos es significativa $\left(x_{2}=2.96 p<0.05\right)$.

Tabla 2.

91 Viños con Crisis Fpilépticas

Recaídas y trastotnos cugnitivos y de: la conducta

$\begin{array}{lllll} & \text { Recaida \% } & \text { No recaida } \% & \text { Total } \\ \text { Con Trastorno } & 1137.9+ & 18 & 62.1 & 29 \\ \text { Sin Tristorno } & 1321.0+ & 49 & 79.0 & 62 \\ \text { Total } & 24 & 67 & 91 \\ & \text { (+) } x=2.96 & p<0.05 & \end{array}$

Electroencefalograma: 30 pacientes tuvieron EEG anormal. De ellos recayeron $10(33,3 \%)$. Un enfermo mostró un foco mixto, 6 tenían actividad paroxistica generalizada lenta, en 2 esta actividad fue irritativa y uno la tuvo mixta. A su vez. en 20 niños, que también presentaron EEG anormal, pero que no recayeron, se encontró que 1 tenfa un foco irritativo, 15 exhibieron actividad paroxística generalizada lenta, 3 la tenian irritativa y uno la tuvo mixta. 40 enfermos no mostraron alteraciones electroencefalográficas, de ellos recayó el $35 \% 14$. Se observa que no existe diferencias entre los niños con EEG anormal y normal con respecto a las recaídas.

\section{DISCUSION}

A pesar que la muestra de la presente investigación ${ }^{1} \mathrm{es}$, a diferencia de las de otros autores, muy homogénea ${ }^{2-3 \cdot 4-5-6-7}$ : parece posible determinar algunos factores que ayudan a predecir las recaídas.

Está demostrado, sin que hasta ahora se haya

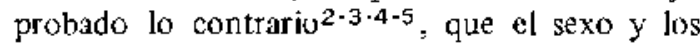
antecedentes de epilepsia en la familia no tienen relación con las recaídas, como ocurre en esta comunicación. Hacen excepción las convulsiones fcbriles $^{8}$, la epilepsia del Pequeño mal ${ }^{9}$ y las crisis epilépticas que ocurren por primera ve $L^{9 \cdot 10}$, en los cuales los antecedentes de epilepsia en la familia son variables de peor pronóstico.

Algunos autores han demostrado que las epilepsias que se inician antes de los dos años de vida ${ }^{4}$, recaen significativamente más que aquellas que comienzan posteriormente. En la presente investigación los grupos etarios se organizaron igual que en dicha publicación ${ }^{4}$ y no fue posible confirmar los hallazgos de esos autores. Al contrario, a pesar que la diferencia no fue significativa, se observó mayor tendencia a recaer en los niños de 3 a 11 años de edad, como también ha sido demostrado por otros investigadores ${ }^{2}$. En otras series de niños ${ }^{3-5}$ tampoco se han encontrado diferencias significativas en la relación de las recaidas con la edad de comienzo, como asimismo en las que incluyen niños y adujtos ${ }^{1.12}$, salvo en una investigación ${ }^{7}$. Es posible que tan contradictorios resultados se deban al tipo de crisis y de epilepsia con que debutaron los pacientes, más que a la edad misma.

A diferencia de las investigaciones emprendidas por la mayoría de los autores, el grupo de nifios estudiados elr este trabajo, no está formado exclusivamente por pacientes epilépticos ${ }^{13}$ sino también por enfermos con crisis únicas y ocasionales. Los enfermos epilépticos presentaron un porcentaje de recaidas $(29,5 \%)$ que no difierc mucho del grupo total $(26,4 \%)^{1}$, ni tampoco del señalado por la mayoría de los autores ${ }^{1}$. No obs. tante dichos pacientes tienen una mayor tenden. 
cia a recaer que aquellos que presentaron una sola crisis antes del tratamiento $(23 \%)$, a pesar que la diferencia no es significativa. Esto último puede tener cierta importancia pues indica que un episodio único, incluso bajo un prolongado tratamiento tiene un $23 \%$ de posibilidades de repetirse, lo cual plantea ciertas dudas acerca de la benignidad de un episodio epiléptico aislado. Probablemente existan algunos factores capaces de predecir las recaidas en dichos enfermos, pero que no fueron estudiados en esta investigación.

Los pacientes con crisis generalizadas tienden a recaer menos ( $25 \%$ ) que aquellos con las liamadas crisis mixtas (generalizadas y focales) $(38,5 \%)$. A pesar de existir una positiva correla. ción, la diferencia no es significativa. Similares resultados han sido comunicados por otros autores $^{4}$. Algunos han podido demostrar, sin embargo, que significativamente las crisis de Gran mal tienen mejor pronóstico que los episodios sicomotores y jacksonianos. Cuando se analizan series en las cuales figuran niños y adultos, en esta perspectiva, los resultados son también contradictorios $2-3-5-7-11-14-15-16$. Ninguno de $\operatorname{los} 3 \mathrm{ni}-$ ños con crisis focales de nuestro estudio recayó. Es un número demasiado pequeño como para extraer conclusiones y la forma electroclinica no fue analizada. Sin embargo no se debe olvidar que ciertos tipos de crisis focales ${ }^{17}$ y algunas formas de epilepsias parciales ${ }^{1 B}$ tienen un buen pro. nóstico.

Los enfermos orgánicos de este trabajo no recaen más que los que no lo son. Otros autores ${ }^{5}$. sin haber definido el concepto de organicidad. han obtenido resultados semejantes. Sin embargo, cuando el criterio de organicidad se restringe a retardo mental, alteración neurológica dependiente de daño de primera neurona ${ }^{3-4}$ o ambos, un significativo mayor número de niños orgáni oos recae. Dentro del grupo de niños orgánicos del presente trabajo hubo 4 con trastornos de aprendizaje específico, sólo 5 tenian retardo mental y 20 exhibian antecedentes de alteración pre, peri o postnatal (algunos tenían más de una caracter ística), y ninguno signos de daño de primera neurona. Si se piensa que los trastornos del aprendizaje son fundamentalmente maduracionales y que la información acerca de alteraciones en relación a la gestación y el parto fue dada verbalmente por las madres, se explica la diferencia entre los resultados aquí presentados y los de otros autores $3 \cdot 4-12$.

Una forma de determinar la severidad de las crisis epilépticas es analizar la frecuencia de los episodios antes de iniciar el tratamiento medicamentoso ${ }^{3-4}$. No se observa una significativa diferencia al dividir el grupo de ninos entre los que tuvieron 20 más crisis de los que presentaron 30 más. Dos investigaciones en niños ${ }^{3-5}$ confirman los hallazgos del presente trabajo y en una de ellas ${ }^{3}$ también fueron excluídos para este análisis las ausencias de Pequeño mal y los espasmos masivos. Otros investigadores ${ }^{7}$, en series que incluyen niños $y$ adultos han obtenido como aqui resultados similares. Sin embargo cuando los niños tienen más de seis crisis de Gran mal previos al tratamiento ${ }^{4}$, recaen significativamente más que aquellos que presentan un menor número de epjsodios, correlación que también ha sido encontrada por otros autores ${ }^{19}$.

No muchos enfermos recaen durante el tratamiento ${ }^{20}$, pero los que vuelven a tener 20 más crisis mientras están recibiendo la medicación, recaen al final de ese período sígnificativamente más que aquellos en los cuales la enfermedad ha sido controlada desde el inicio de la terapia. De hecho, hay consenso en la literatura ${ }^{3-4}$ que cuando esta eventualidad ocurre, el pronóstico del cuadro en relación a las recaidas es más malo.

Los nifios epilépticos presentan en porcentajes que van del 9 al 33\% trastornos de tipo psiquiátrico $^{21}$ y de acuerdo a algunos autores ${ }^{22}$, en dichos pacientes el control de su crisis es dificil y el no cumplimiento es frecuente ${ }^{2 \mathbf{3}}$. No es extraño entonces, como ha sido demostrado en esta investigación que los pacientes con trastornos cognitivos y de la conducta recaigan significativamente más que aquellos que no los tienen.

Sorprendente resulta sin embargo, la ausencia en la literatura de alusiones en relación a esta variable. Los resultados obtenidos aqui no pueden ser considerados como definitivos, pues el grupo de niños portadores de dichos trastornos, resultó ser muy heterogéneo y por que además es imposible determinar con precisión si las alteraciones cognitivas y de la conducta de los pacientes se debjeron o no al efecto de los anticonvulsivantes $23-24-25 \cdot 26$

El EEG en los niños está estrechamente ligado al estado maduracional de su sistema nervioso central y su expresión correlacionada con la edad ${ }^{27}$. Estos y otros factores deben ser tomados en cuenta al analizar el EEG en $\operatorname{los}$ niños ${ }^{28}$. Por otra parte, el número de EEG y las condiciones en las que se realiza el examen (sueño, vigilia y métodos de provocación $)^{27-28}$ son variables que influyen en el resultado. Teniendo en mente estas conside. raciones, podria ser explicable la falta de diferencia significativa encontrada en la presente publicación entre anormalidad electroencefalográfica y recaídas, hecho que también ha sido mencionado por otros autores ${ }^{3}$. También en adultos esta ausencia de correlación ha sido comunica$\mathrm{da}^{15-29}$. Sin embargo, otros investigadores han demostrado que la actividad irritativa y las lenti. tudes paroxísticas incrementan en forma signifi- 
cativa las probabilidades de recaida cuando ocurren esas alteraciones del EEG al final del tratamiento ${ }^{4.5}$, así como también cuando se observa un franco empeoramiento de los ritmos eléctricos del paciente antes de suprimir la medicación ${ }^{30}$. A pesar de no existir unanimidad, la opinión generalizada es que las alteraciones focales y los paroxismos irritativos son importantes predictores de recaídas ${ }^{4-5-6-11 \cdot 14-17-30-31-32}$.

Como se ha apreciado a lo largo de esta discusión, no hay acuerdo entre los diversos autores acerca de cuales son los factores de pronóstico en los niños con crisis epilépticas, sean éstos significativos o simples correlaciones. Es probable que este hecho esté en relación a que las poblaciones estudiadas y la metodología empleada son dife. rentes. Es posible también que no sea el estudio de variables aisladas sino el análisis multifactorial, el que dé la clave para predecir las recaidas, pero tampoco hay unanimidad cuando dicha metodo. logía es empleada ${ }^{3-4}$.

La decisión de suspender la medicación a un paciente está naturalmente basada en las características propias del enfermo, pero se debería tener presente que la longitud del tratamiento no parece estar relacionado con las recaidas ${ }^{i}$, que existe un alto número de niños chilenos con epilepsia $(2.7 \%)^{33}$, que el tratamiento tiene un costo económico y social ${ }^{34}$ y que los medicamentos no son inocuos $35-36-37$.

Por lo anterior y porque algunos enfermos ticnen factores significativamente predictores de recaídas y otros pacientes no los tienen, es que sería aconsejable no tratar a todos los enfermos por igual tiempo, sino que aquellos con bajo riesgo de recaidas deberian recibir medicación por lapsos más breves ${ }^{4-38}$, que los generałmente recomendados.

\section{RESUMEN}

En un grupo de niños con crisis epilépticas se estudian los posibles factores predictivos de recaídas. Se encontró que aquellos que durante el tratamiento repiten dos o más crisis y aquellos con rastornos cognitivos y de la conducta recaen con mayor frecuencia ел un porcentaje estadistjcamente significativo en relación a los que no acusan estos problemas.

Otras caracteristicas aparentemente no influirían en la incidencia de las recaídas. Ejemplo de éstos son: edad, sexo y antecedente familiar de epilepsia, lo que concuerda con el hallazgo de otros autores.

Un tercer grupo de factores (incluídas las crisis mixtas, y epilepsia) se asoció a ила mayor frecuencia de recaídas, pero dicha correlación, a diferencia de lo comunicado por otros autores, no resultó significativa.

\section{REFERENCLAS}

'Devilat, M.: Gennain, L, y Valenzuela, B.: Pionóstico a largo plazo en 91 nirios con erisis epilépticas tratados por $\$$ años. Rev. Chil. Pediat. Vol. 54 (5) 1983. (En Prensa).

${ }^{2}$ Hollowach, J., Thurston, D.L. and OLeary, J.: Prognosis in childhood epilepsy. Follow-up study of 148 cases in wich therapy had been suspended after prolonged anticonvulsant control. New England J. Med. 286: 169, 1972.

${ }^{3}$ Hollowach, J., Thurston, D. L., Hixon, B. B. and Keller, A. J.: Prognosis in childhood epilepsy. Aditional follow-up of 148 chiddren 15 to 23 years after withdrawal of anticonvulsant therapy. Ncw Engl. J. Med. 306: 381, 1982.

4 Emerson, R. D'Souza, B J., Ying, E. P., Holden. K. $R$., Mellits, $E$. $D$. and Freeman, $S$. $M$. Stopping medication in children with epilepsy. Predictors of outcome. New Engl. J. Med. 304: 1125, 1981.

${ }^{5}$ Groh, C.: Zur Frage der Heilbarkeit Kindicher Epilepsien. Wien Klin. Wochenschr. 87: 1, 1975.

6 Oller-Daurella, L, Oller F-V, L. and Pamies, R.: Clinical therapeutic and social status of epileptic patient without seizures for more than five years. In: Penry, J.K. (Ed.): Epilepsy, The Eighth Intemational Sympoxium. Raven, New York, 1977, pp. 69.

7 Janz, D.: In tema di remisione e di ricomparsa di ctisi durante e dopo il trattamento farmacologico dell'epilessia. Boll. Lega It. Epil. 39: 95, 1982.

8 Netson, K. B. and Ellenberg, J. H.: Prognosis in children with febrile seizures. Pediatrics 6l: 720 , 1978.

9 Sato, S., Dreifuss, F.E, and Penry, J. K.: Pjognosis factors in absence sizurcs. Neurology 26: 788, 1976.

10 Hauser, w. A., Anderson, V. E. and Loewenson, $R$. $B .:$ Scizure recurrence after a first unprovoked seizure. New Engl. J. Med. 307; 522, 1982.

11 Jut-Jenser, $P$.: Frcquency of recurrence after discontinuance of anticonvulsant therapy in patients with epilec tic scizures. Epilepsia S: 532, 1964

12 Annegers, J.K., Houser, W.A. and Elvebock, L.R.: Remission of seizures and relapse in patients with cpilepsy. Epilepsia 20: 729, 1979.

13 Gastaut, H.: Diccionario de epilepsia. Parte I: Definiciones. Organización Mundial de la Salud, Suiza, 1973. pp. 47.

14 Oller-Datrelita, L. and Marquez, J.: Survey of 100 cpilepties who have not seizures for ten years or more. Epilepsia 13: 161, 1972.

Is Jonz, D. and Sommer-Burkhardt, E. M.: Discontinuation of antiepileptic drugs in patients witlt epilepsy who lave been seizure-ftee for more than two years. In: Janz, D. (Ed.) Epilcptology. Thieme Raven Press, New Yotk, 1977, pp. 228.

16 Janz, D. Kern. A., Mossinger. H. J. und Puhlmann. $U_{\text {: }}$ Rückfall- Prognose nach Reduktion der Medikamente bei Epilepsiebelyandlung. In: Remschmidt, H., Renty, R, und Jungmann, J. (eds.): Epilepsie, 1981 . (Im Verkag).

17 Roger, J., Dravet, C., Menéndez, P. et Burecu, M.: Les spilepsies partielles de L'enfart - evolution et facteurs de pronostic. Rev. E.E.G. Neurophysiol. 11: 431, 1981 
16 Heycoop ten Ham, M. W.: Complete recovery from epilepsy? Huisarts en wetenshap. 23: 309, 1980.

19 Todf, K.: Zu Spätprognose Kindlicher Epilepsie Ergebnisse einer prospektiven Längsschnittsstudie. Dtsch. Gesundh Wesen 36: 2012, 1981.

20 Devilat, M. y Taha. L.: Epilepsia infantil criptogenética. I. Aspectos clínicos y asistenciales, electroencefalografía $y$ tratamiento. Pediatría (Santiago) 21: 204,1978 .

21 Devilat, $M$, y Taha, L.: Epilepsia infantil criptogenética. II. Rendimiento escolar y aspectos psìquiátricos. Pediatría (Santiago) 21: 208, 1978.

22 Holdsworth, $L$ and whimore, $K .:$ A stud of children with epilepsy attending ordinary schools. I, Their seizure patterns, progress and behaviour in school. Develop. Med. Child. Neurol, 16: 746, 1974.

23 Camfield, C.S., Chaplin, S. Doyle, A., Shapiro. S. J., Cummings, $C$. and Camfield, $P$. R.: Side effects of phenobarbital in toddlers: behavioral and cognitive aspects. J. Pediatr. 95:361, 1979.

24 Trimble, $M$. and Corbett, $J$, : Anticonvuisant drugs and cognitive function. In: Wada, J.A. and Penry, J.K. (eds.): Adyances in Epileptology. Raven Press, New York, 1980, pp. 113.

25 Trimble, $M$. Thompson, $P, J$, and Huppert, $F$ : Anticonvulsant drugs and cognitive abilities. In: Canger, R., Argeleri, F, and Penry, J.K. (eds.): Advances in Epileptology Raven Press, New York, 1980, pp. 199.

26 Marzuca, N.: Efectos de los anticonvulsivantes sobre el coeficiente intelectual. Servicio de Neurologia y Psiquiatria. Hospital Luis Calvo Mackenna. Santiago de Chile. (Comunicación personal) 1982.

27 Tsuboi, T.: Correlation between EEC abnormality and age in childhood. Neuropädiatrie 9: 229, 1978.

2B Dumermuth, G.: Elektroencepfalographie in Kindesalter. Thieme, Stutteart, 1976, S. 20.

29 Overweg, J., Rowan, A.J., Binnie, C.D. Oosting, J. and Nagelkerke, N.J.D.: Prediction of seizure recu- rrence after withdrawal of antiepileptic drugs. In: Dam, M., Gram., L. and Perry, J.K. (eds.): Advances in Epileptology. Raven Press, New York, 1981, Pp. 503.

${ }^{30}$ Foerster, Ch. un Schmidberger, D.: Prognostische Wertigkeit von EEG-Verlaufsuntersuchungen bei Kindern mit Anfallsrezidiven nach Absetzen der antikonvulsiven Therapie. In: Doose, He und GrossSelbeck, G. (eds.): Epilepsie 1978. Thiome, Stuttgart, 1979, S. 252.

31 Strobos, R. R. J.: Prognosis in convulsiye disorders. Arch. Neurol. (Chicago), 1: 216, 1959.

${ }^{32}$ Rowan, A. J., Overweg, J., Sadikoghu, S., Binnie, C. D., Nagelkerke, N. J. D. and Hunteler, E.: Seizure prognosis in long-stay mentally subnomal epileptic patients: interrater EEG and clinical studies. Epilepsia $21: 19,1980$.

${ }^{33}$ Ciofalo, N., Kirschbaum, A, Fuentes, A., Cordero,

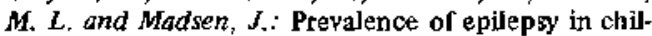
dren of Melipilla, Chile. Epilepsia 20: 261, 1979.

34 Devilat, M., Germain, L. y Valenzuela, B.: Seguimiento a mediano plazo de niños tratados por 5 años por convulsiones. Reunión mensual de la Sociedad de Psiquiatría y Neurología de la Infancia y Adolescencia. Santiago de Chile 2 de Junio de 1983.

${ }^{35}$ Reynolds, E. H.: Chronjc antiepileptic toxicity: a review Epilepsia 16: 319, 1975.

36 Rating. D., Jaeger, E., Pattberg, B, Engelke, K., Bekcmannagetto, $G$. und Helge, $H$. Antiepileptika wàhrend der Schwangerschaft und Kindesentwicklung, In: Doose, H. und Gross-Selbeck, G. (edis): Epilepsie 1978. Thieme, Stuttgart, 1979. S. 240.

37 Janz, D.: Uber das Risiko von Missbildungen und Entwicklungsstörungen bei $\mathrm{K}$ indem von Eltem $\mathrm{mit}$ Epilepsie. Nervenarzt 50: 555, 1979.

36 Gordon, N.: Duration of treatment for childhood epilepsy. Develop. Med. Child Neural. 24: 84, 1982. 